\title{
Kyphoplasty in the Early Oncologic Diagnosis and Treatment of Vertebral Fractures: A Clinical Study
}

\author{
Yener AKYUVA ${ }^{1}$, Benan BAYSOY ${ }^{1}$, Eyup Can SAVRUNLU ${ }^{1}$, Gulseli Berivan SEZEN ${ }^{1}$, Osman BOYALI ${ }^{1}$, Halil CAN²*, \\ Erdinc CIVELEK ${ }^{1}$, Serdar KABATAS ${ }^{1}$
}

1University of Health Sciences, Gaziosmanpasa Training and Research Hospital, Department of Neurosurgery, Istanbul, Turkey
Biruni University, School of Medicine, Department of Neurosurgery, Istanbul, Turkey
This study has been presented at the Aging Spine Symposium between 31 October and 03 November 2019 at Antalya, Turkey

Corresponding author: Yener AKYUVA yenerakyuva@hotmail.com

\section{ABSTRACT}

AIM: To elucidate the characteristics of kyphoplasty in correlation with spinal metastasis.

MATERIAL and METHODS: Data of patients treated by kyphoplasty between January 2017 and December 2019 were reviewed retrospectively. Preoperative prophylactic antibiotics and low-molecular-weight heparin injections were performed. Postoperative follow-up was conducted at least 24 hours after the procedure. All patients were treated under sedoanalgesia. Bone biopsies were collected from all patients.

RESULTS: One hundred ninety-nine vertebra fractures were treated in 130 patients. The mean age of the patients was $65.27 \pm 8.79$ years (18-90 years) and 66 patients were male (50.7\%). Forty-five patients had osteoporosis, six patients showed malignancy, and osteomyelitis was found in three patients, while the others' presentations were secondary to trauma. Most commonly, the $L 1$ ( $n=59$ ), Th12 ( $n=45)$, and L2 ( $n=34)$ levels were found to develop vertebral fractures. Forty patients had multiple levels of vertebral fracture, with a higher rate of osteoporosis $(n=24 ; 60 \%)$. Three patients showed undiagnosed oncologic disease with an initial diagnosis of acute fracture following minor trauma, while the primary oncologic diagnosis was established by bone biopsy taken during the routine procedure in each procedure (e.g., plasmacytoma, lymphoma, adenocarcinoma of the lung). None of the patients developed an infection due to kyphoplasty, permanent neuromotor deficit, or mortality. The mean postoperative hospital length of stay was 1.6 days.

CONCLUSION: Bone biopsy should be performed to diagnose early spinal metastases. Although an accurate bone biopsy may not be obtained from some patients, particularly from those with osteoporosis, performing bone biopsy during the procedure does not cause time loss or any other complications, and protects the surgeon from possible medicolegal problems.

KEYWORDS: Kyphoplasty, Bone biopsy, Spinal metastases, Vertebral fracture

ABBREVIATIONS: CT: Computed tomography, DEXA: Dual-energy X-ray absorptiometry, LMWH: Low-molecular-weight heparin, MRI: Magnetic resonance imaging, PBK: Percutaneous balloon kyphoplasty, STIR: Short-time inversion-recovery, TLICS: Thoracolumbar injury classification and severity, VCF: Vertebral compression fracture.

Yener AKYUVA (D) : 0000-0002-2424-3117

Benan BAYSOY (1): 0000-0001-9536-3768

Eyup Can SAVRUNLU (1) : 0000-0001-9022-200X
Gulseli Berivan SEZEN (1) : 0000-0001-9129-5470

Osman BOYALI (D): 0000-0002-2500-1718

Halil CAN (D) : 0000-0002-5699-4089
Erdinc CIVELEK (1) : 0000-0003-2691-6861 Serdar KABATAS (D) : 0000-0002-3988-4064 


\section{INTRODUCTION}

$\mathrm{V}$ ertebral compression fracture (VCF) is a common occurrence and often found as a consequence of trauma, osteoporosis, or vertebral metastasis of malignant tumors (3). It is a condition that has a negative impact on both quality of life and physical function. Symptomatic VCFs can promote increased morbidity rates with conditions including severe low back pain, increased thoracic kyphosis, decreased pulmonary function, sleep disturbance, vertebral height loss, and impaired quality of life (5).

Percutaneous balloon kyphoplasty (PBK) is a minimally invasive treatment option used for the management of symptomatic VCF that facilitates the stabilization of the spine and promotes pain relief through injecting a biomaterial [usually polymethyl methacrylate (PMMA)] percutaneously (21). Also, PBK supports vertebral height restoration and sagittal balance regulation following balloon expansion of the fractured vertebra. PBK has become widely used to address backbone and spine metastasis of neoplastic disease and osteoporotic VCFs worldwide $(2,13)$.

Symptomatic spinal metastasis develops in approximately $30 \%$ of individuals diagnosed with cancer (17). In recent years, new treatment methods for cancer patients that prolong life have also brought about the expectation of an increase in the quality of life. Metastatic vertebral involvement causes erosion of the vertebra, which may result in VCF followed by pain and spinal instability (13). Especially, malignancies in the prostate, breast, blood, and lungs can cause primary organ pathology as well as vertebral metastasis (18). Damage caused by cancer in the vertebra corpus may begin to effectuate clinical symptoms even following only minor trauma. It is known that malignancy incidentally might be diagnosed at a rate of $1 \%$ to $3 \%$ from biopsies taken during PBK. The clinician should be careful when establishing the diagnosis of VCF, whether spontaneous or secondary to an insidious pathology such as cancer (15).

In this study, the clinical and radiographic results of patients who underwent PBK treatment at a single health center were evaluated. As part of this research, we wanted to emphasize the degree of bone biopsy's importance during the PBK procedure among patients who did not exhibit any oncological disease history and also had a first diagnosis of acute VCF after minor trauma.

\section{MATERIAL and METHODS}

We retrospectively reviewed the data of patients treated by PBK in the neurosurgery clinic between January 2017 and December 2019 with a diagnosis of VCF. These patients were referred to the emergency service with spinal trauma and, during physical examinations, the patients showed thoracolumbar pain. Among these patients, computed tomography (CT) imaging was performed in affected and suspected spinal regions. Short-time inversion-recovery (STIR) sequencing in thoracolumbar magnetic resonance imaging (MRI) was applied in those patients who demonstrated VCF during CT imaging. STIR-sequence MRI results led patients with hyper- intensities supporting acute VCF to be admitted to the neurosurgery service. The Thoracolumbar Injury Classification and Severity (TLICS) score of each patient was established. Finally, patients with a TLICS score of four points or less underwent preoperative anesthesia for PBK treatment.

Before the operation, prophylactic antibiotic treatment and lowmolecular-weight heparin (LMWH) injection were conducted due to the risk of embolism. All patients were managed under sedoanalgesia and standard surgical procedures. Continuous two-way fluoroscopy was performed during PBK and a PMMA injection in a slow and controlled fashion was completed. The patient's neurological examination was repeated before, during, and immediately after PBK. After the patient was removed from the operating room, early postoperative thoracolumbar CT imaging was performed and resultant scans were evaluated for cement leakage. Patients were followed up with for at least 24 hours postoperatively and then discharged.

The technique adopted in this study involved the insertion of inflatable balloon tamps, percutaneously to the vertebrae and which, through a gradual inflation process, created a cavity within the fractured or collapsed vertebral body. When the vertebra corpus was reached, a bone biopsy was gathered. Then, the injection of PMMA into the vertebral body was done to enhance the stabilization and strength of the fracture and promote a reduction in kyphotic deformity, achieving an almost-immediate analgesic effect $(3,15)$.

Permission to use the retrospective data was obtained from the chief physician of the hospital as well as from the chairman of the neurosurgery department. Our study was planned in accordance with the Declaration of Helsinki.

\section{Statistical Analysis}

The Statistical Package for the Social Sciences version 21.0 software program (IBM Corp., Armonk, NY, USA) was used for statistical analysis. Continuous data were summarized as means \pm standard deviations, while categorical data were summarized as numbers and percentages. The chi-squared $\left(x^{2}\right)$ test was used to evaluate two independent categorical groups for comparisons between groups. Statistical significance was noted as $\mathrm{p}<0.05$.

\section{RESULTS}

In our clinic, 199 VCFs were treated in 130 patients. The mean age of patients was $65.27 \pm 8.79$ years (18-90 years) and 66 patients were male $(50.7 \%)$ and 64 patients were female (49.3\%). Regarding the cause of VCF, osteoporosis was diagnosed in 45 patients, malignancy was diagnosed in six patients, and osteomyelitis was found in three patients, while the remaining cases were secondary to trauma. Most commonly, the lumbar (L)1, $(n=59)$, thoracic $(T h) 12(n=45)$, and L2 $(n=34)$ levels were found to develop VCFs. Among the 40 patients with osteoporosis, $24(60 \%)$ had multilevel VCF. Ninety-one (70\%) patients had single-level VCFs, 19 patients had double-level VCFs, 12 patients had tri-level fractures, and eight had quad-level fractures. Fifteen patients had experienced previous VCFs, while four patients had a 
history of posterior instrumentation in the lumbar region. None of the patients in this study developed an infection due to PBK, permanent neuromotor deficit, or mortality. The mean postoperative hospital length of stay was 1.6 days. Posterior instrumentation was performed after kyphotic deformities were detected during the clinical and radiological follow-up of three patients. One patient developed cauda equina syndrome due to cement overflowing into the spinal canal and underwent emergency decompression surgery. In total, only four patients required posterior instrumentation (2\%). PBK was performed unilaterally to 93 vertebrae and bilaterally to 106 vertebrae. Dural injury and spinal subdural hematoma due to the medial passage of kyphoplasty cannula were observed in two patients. PMMA migration to the vena cava was found in two cases, while that to the intervertebral disc was found in eight patients. All patients received 2- to 6-cc cement injections per vertebra during the procedure. The procedure was terminated when the vertebral corpus was filled with enough PMMA or a PMMA leak outside the corpus was suspected.

There was no known oncological disease in three patients and the initial diagnosis was acute VCF following minor trauma, whereas primary oncologic diagnosis was determined by bone biopsy (e.g., plasmacytoma, lymphoma, lung cancer metastasis) (2.3\%). A 54-year-old female patient was admitted to the emergency service after falling in the bathroom. The patient, whose neurological examination was normal, had only back pain. L3 VCF was diagnosed on a lumbar CT scan and the STIR-sequence MRI findings were compatible with acute VCF (Figure 1A-F). In the radiological evaluation, no other pathology was found and PBK treatment was performed. Biopsy results suggested the presence of nonHodgkin's lymphoma metastasis. Separately, a 63-year-old female patient applied to the emergency department with severe back pain after carrying a load. Lumbar CT and MRI revealed L2 and L4 acute VCFs (Figure $2 A-I)$. The presurgery diagnosis of the patient was osteoporosis but biopsy results were compatible with plasmacytoma. In addition, a 51-yearold male patient came to the emergency service after falling from a height of approximately $1 \mathrm{~m}$ and reported severe pain in his back. Th9 acute VCF was detected in the patient with normal neurological examination results. At the same time, the patient had minimal VCFs in the Th11 and L1 vertebrae. MRI suggested potential metastatic involvement (Figure 3A$F)$. The patient was operated on and bone biopsy revealed metastasis of adenocarcinoma of the lung. Finally, three patients received previous oncological diagnoses and the presence of spinal metastasis was confirmed by biopsy (i.e., multiple myeloma, lung cancer). Thirteen (10\%) patient pathology reports indicated only acellular bone or insufficient tissue were available.
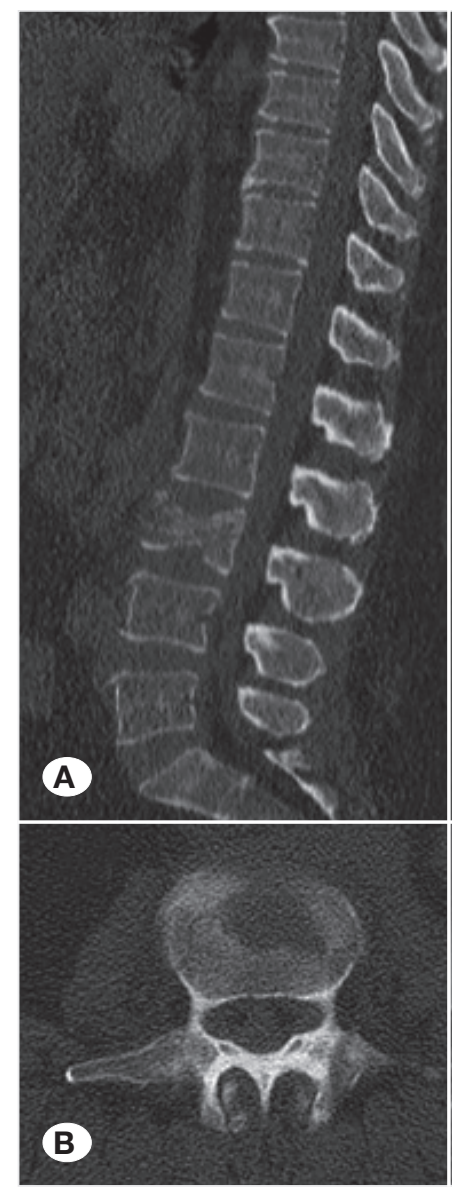
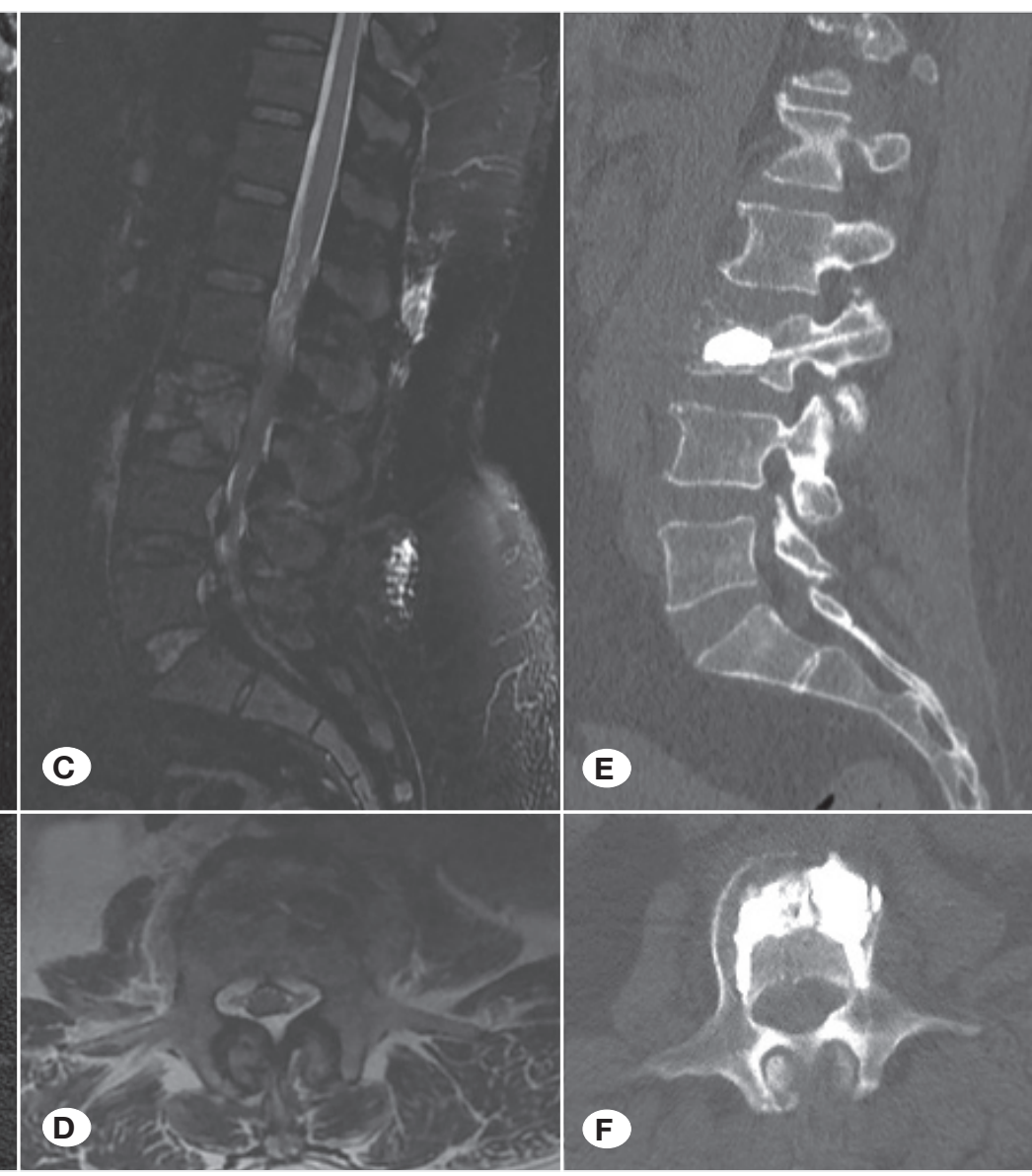

Figure 1: L3 VCF

was diagnosed in the sagittal section (A) and axial section (B) via thoracolumbar computed tomography (CT). Axial and sagittal cross-section shorttime inversionrecovery sequence lumbar magnetic resonance imaging revealed edemarelated hyperintensity after acute trauma in the vertebral corpus (C, D). Axial and sagittal section CT view after L3 kyphoplasty treatment using the bilateral approach (E, F). 


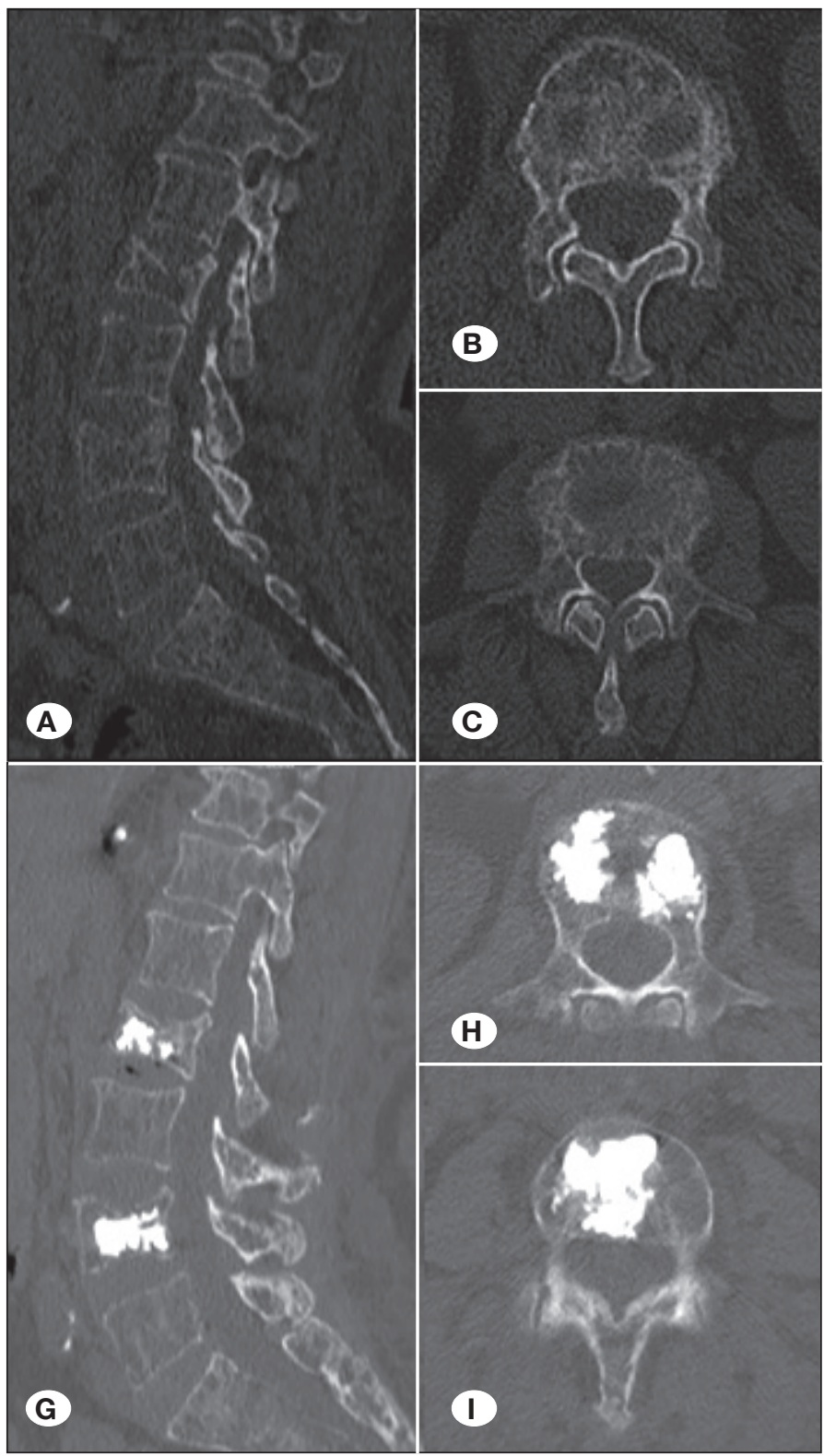

\section{DISCUSSION}

PBK and vertebroplasty are effective surgical techniques that are increasingly receiving attention (2). To provide pain relief and prevent deformity in the context of VCF, a strengthening intervention should be performed (3). The vertebral corpus can be reached with a cannula of the PBK and PMMA is injected into the corpus to stabilize the fracture $(3,15)$. Unlike during vertebroplasty, a cavity is formed in the process of PBK (2). A balloon is then inflated inside the corpus, the collapsed vertebra height is increased, and PMMA is injected inside the cavity. As such, the height of the vertebral corpus is restored after treatment $(2,3)$. These two techniques have largely replaced open surgery in stable cases because they are less invasive, lead to less complications, and have better positive effects on morbidity and mortality (6). PBK in particular is

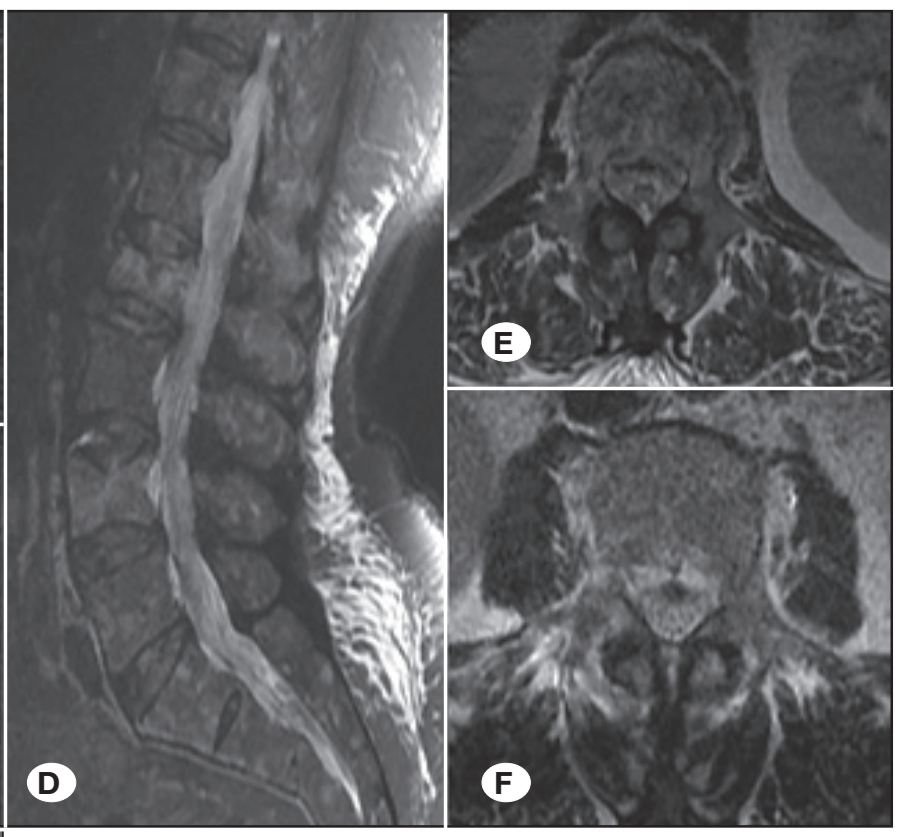

Figure 2: L2 and L4 VCF was diagnosed in the sagittal section (A), L2 (B) and L4 (C) axial section via thoracolumbar comouted tomography (CT). Axial and sagittal cross-section short-time inversion-recovery sequence lumbar magnetic resonance imaging revealed edema-related hyperintensity after acute trauma in the vertebral corpus (D-F). Axial and sagittal section CT view after L2-L4 kyphoplasty treatment using the bilateral approach (G-I). preferred by our clinic as a priority treatment in patients with VCF.

It is not yet known exactly how pain relief occurs with PBK. The reduction in pain may be due to mechanical stabilization achieved by bone cement injection. At the same time, it is thought that the heat effect occurring during the polymerization phase of cement is effective through the pain-transmitting receptors in the bone due to causing localized nerve cell death in the bone and/or nerve endings. The heat effect of polymerization in vertebral metastases also may prevent the development or cause the death of cancer cells $(3,7)$. Therefore, the conduct of PBK in cases of spinal metastasis is becoming more and more common.

CT may not provide entirely accurate information about whether the VCF is acute or chronic (1). Therefore, MRI is more 

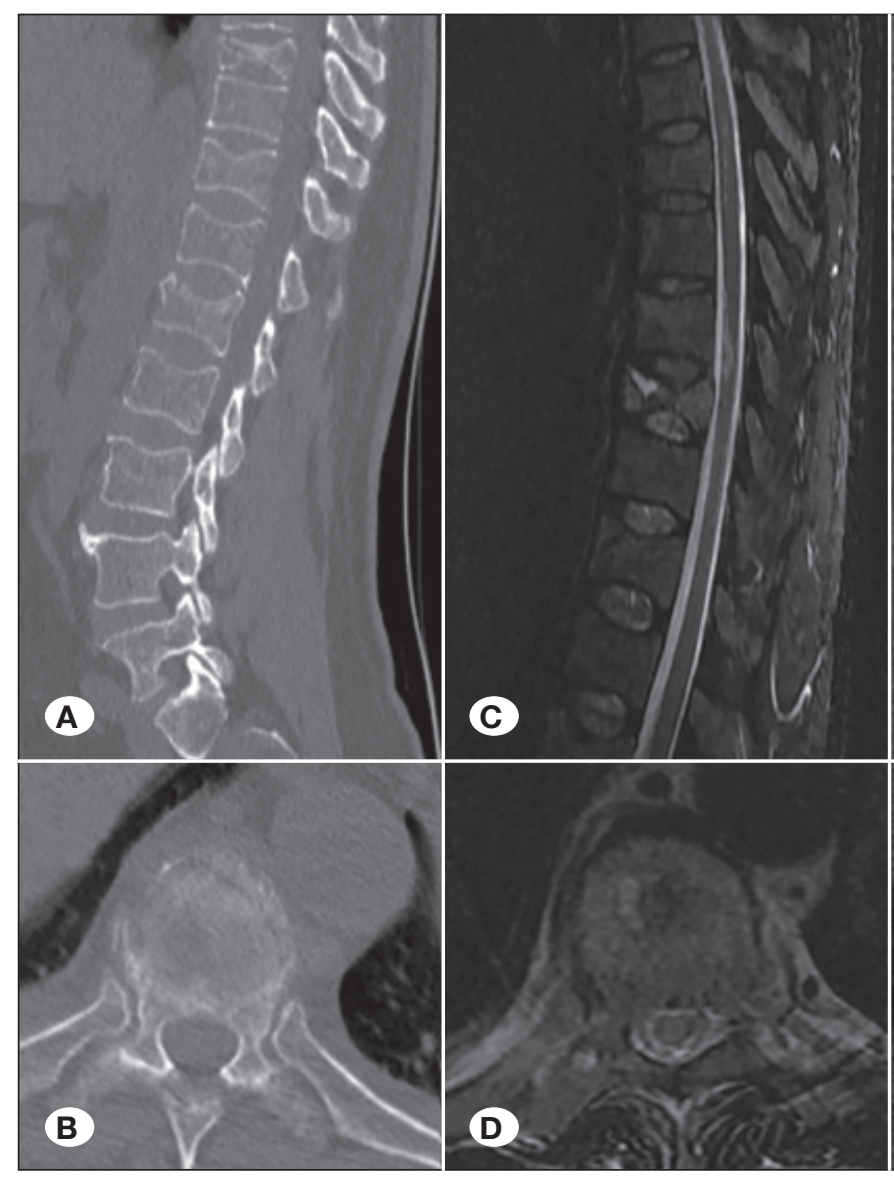

(E)
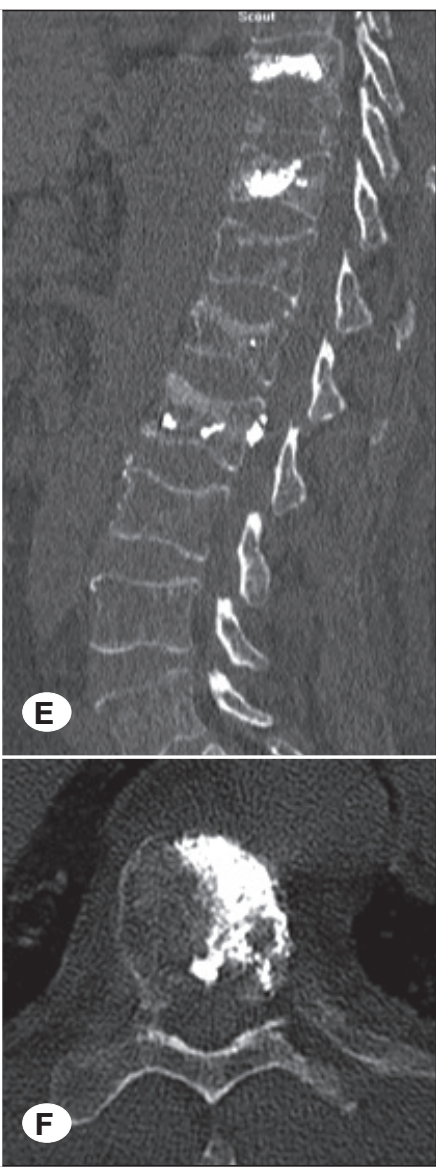

Figure 3: Th9, Th11, and L1 VCF was diagnosed in the sagittal section (A) and Th9 axial section (B) via thoracolumbar computed tomography (CT). Axial and sagittal cross-section short-time inversion-recovery sequence lumbar magnetic resonance imaging revealed edema-related hyperintensity after acute trauma in the Th9 vertebral corpus (C, D). Axial and sagittal section CT view after Th9 kyphoplasty treatment using the unilateral approach (E, F). effective in the diagnosis of acute VCFs. The development of edema-induced hyperintensity in the vertebra, especially in STIR-sequence MRI, supports a diagnosis of acute VCF. If a patient is suspected to have spinal metastasis, contrastenhanced MRI should be performed (11). Unfortunately, in some cases, a definitive diagnosis cannot be made despite all these tests. Jung et al. reported that MRI cannot always distinguish between acute VCFs and metastatic fractures (8). Therefore, the gold-standard method for differential diagnosis is pathological examination.

In particular, malignancies of the lungs, breast, prostate, and blood are often capable of spinal metastasis without clinical presentation. Tumors are typically located in the vertebra and exhibit an osteolytic capacity $(17,18)$. Such a deformity in the vertebra affects the spine dynamics negatively and may provoke VCF, which may cause clinical problems even in the context of minor trauma $(13,15)$. In such a case, radiological and clinical results may be consistent with isolated VCF $(8,11)$. In the literature, rates of incidental spinal metastasis detection during PBK are between $1 \%$ and $3 \%(13,15,20)$. In our clinical experience, the incidence of incidental oncologic diagnosis from biopsy was $2.3 \%$.

Dalbayrak et al. reported that performing PBK in patients with spinal metastasis improved both the spinal deformity of the patient and their visual analog scale score. These authors also noted that this treatment, together with advances in oncologic treatments, reduced the risks for morbidity and mortality (4). Pflugmacher et al. also found that patients with spinal metastasis tolerated PBK (16). Meanwhile, Dalbayrak et al. reported a statistically significant improvement following PBK in pain severity, vertebral body height, and segmental kyphosis (4). Three patients in our clinical series were diagnosed with spinal metastasis from bone biopsy, while three patients were known to have such before biopsy. The positive effects of PBK previously reported in the literature were observed in these six patients.

It has also been suggested in the literature that conducting bone biopsy during PBK treatment does not increase the possibility of complications. Ozger et al. emphasized that taking a small bone biopsy during the treatment did not increase the likelihood of complications and did not cause a serious loss of operative time. Such will also enable the patient to receive early oncological treatment (15). In our study, the oncologic treatment of three patients was started earlier due to the results of biopsies taken during the procedure.

When faced with VCF, many clinicians may hypothesize the cause to be isolated trauma. In such a case, the lack of biopsy during treatment may constitute a medicolegal problem. Recent studies have shown that malpractice cases against physicians have increased in frequency. Mukherjee et al. 
Akyuva Y. et al: Diagnosis of Spinal Metastasis from Kyphoplasty

reported on the specific increase of neurosurgical medicolegal cases (12). Nowak et al. suggested that the incidence of positive histology in PBK is high and, if no biopsy is taken, malpractice will be alleged (14). In our study, the biopsy results of three patients were positive and the filing of a possible malpractice suit was prevented. Of note, in our series, 13 pathology reports included insufficient material; however, this is consistent with the literature (15), and is also legally safe for the clinician because of the biopsies (14).

The coexistence of VCFs of multiple levels is high especially in the case of osteoporosis and oncologic metastasis (19). In our study, $60 \%$ of VCFs of multiple levels were detected in patients with osteoporosis, while four patients with oncologic spinal metastasis had VCFs of multiple levels. Therefore, if multiple VCFs are detected, spinal metastasis should be kept in mind $(3,9,19)$. Kurra et al. found that VCFs in osteolytic or osteoporotic vertebrae exhibited high rates of affecting multiple levels (9). Mehta and Sebro reported that dualenergy X-ray absorptiometry (DEXA) might reveal incidental osteoblastic osseous metastases. Clinicians can benefit from the use of DEXA in the distinction between osteoporosis and metastasis (10).

In our clinical series, no cases of permanent mortality or morbidity due to PBK treatment developed. Patients were given $1 \mathrm{~g}$ of cefazolin prophylactically and LMWH prior to the procedure and mobilized early after surgery. Neither thrombosis nor surgical-site infections were observed in our study population. The involvement of continuous two-way scope imaging, a smooth and controlled injection protocol, and standard surgical procedures make this treatment more reliable.

\section{CONCLUSION}

Routine bone biopsy should be performed during PBK. No matter how small the material is that can be removed during the procedure, a pathological examination should be performed. Compliance with standard surgical rules, the provision of prophylactic antibiotics and $\mathrm{LMWH}$, the involvement of continuous fluoroscopy guidance during bone cement application, and the completion of slow and controlled injections are ideal means to achieve successful procedural outcomes. PBK is both a therapeutic and diagnostic method that can improve patient life expectations.

\section{ACKNOWLEDGEMENTS}

The Turkish Neurosurgical Society partly supported the preparation of this article for publication.

The addresses for the author with asterisk(s) are as follows:

*Medicine Hospital, Neurosurgery Clinic, Istanbul, Turkey.

\section{REFERENCES}

1. Brant-Zawadzki M, Miller EM, Federle MP: CT in the evaluation of spine trauma. Am J Roentgenol 136(2): 369-375, 1981
2. Cagli S, Isik HS, Zileli M: Vertebroplasty and kyphoplasty under local anesthesia: Review of 91 patients. Turk Neurosurg 20(4):464-469, 2010

3. Civelek E, Cansever T, Yilmaz C, Kabatas S, Gulsen S, Aydemir $\mathrm{F}$, Caner $\mathrm{H}$ : The retrospective analysis of the effect of balloon kyphoplasty to the adjacent-segment fracture in 171 patients. J Spinal Disord Tech 27(2):98-104, 2014

4. Dalbayrak S, Onen MR, Yilmaz M, Naderi S: Clinical and radiographic results of balloon kyphoplasty for treatment of vertebral body metastases and multiple myelomas. J Clin Neurosci 17(2):219-224, 2010

5. Edidin AA, Ong KL, Lau E, Kurtz SM: Morbidity and mortality after vertebral fractures: comparison of vertebral augmentation and nonoperative management in the Medicare population. Spine 40(15):1228-1241, 2015

6. Gill JB, Kuper M, Chin PC, Zhang Y, Schutt JR: Comparing pain reduction following kyphoplasty and vertebroplasty for osteoporotic vertebral compression fractures. Pain Physi 10(4):583-590, 2007

7. Jefferiss CD, Lee AJC, Ling RSM: Thermal aspects of selfcuring polymethylmethacrylate. J Bone \& Joint Surg 57(4): 511-518, 1975

8. Jung HS, Jee WH, McCauley TR, Ha KY, Choi KH: Discrimination of metastatic from acute osteoporotic compression spinal fractures with MR imaging. Radiographics 23(1):179-187, 2003

9. Kurra S, Metkar U, Lieberman IH, Lavelle WF: The effect of kyphoplasty on mortality in symptomatic vertebral compression fractures: A review. Int J Spine Surg 12(5):543-548, 2018

10. Mehta SD, Sebro R: Random forest classifiers aid in the detection of incidental osteoblastic osseous metastases in DEXA studies. Int J Comput Assist Radiol Surg 14(5):903-909, 2019

11. Meyers SP, Wiener SN: Magnetic resonance imaging features of fractures using the short tau inversion recovery (STIR) sequence: Correlation with radiographic findings. Skeletal Radiol 20(7): 499-507, 1991

12. Mukherjee $S$, Pringle $C$, Crocker $M$ : A nine-year review of medicolegal claims in neurosurgery. Ann R Coll Surg Engl 96(4):266-270, 2014

13. Noriega DC, Krueger A, Ramajo RH, Ardura F, Munoz M, Sahin $S$ : Long-term benefits of percutaneous anatomical restoration of vertebral compression fractures linked to malignancy. Turk Neurosurg 26(4):608-614, 2016

14. Nowak S, Müller J, Schroeder HW, Müller JU: Incidence of unexpected positive histology in kyphoplasty. Eur Spine J 27(4):847-850, 2018

15. Ozger O, Kaplan N: Clinical results of percutaneous vertebroplasty in thoracolumbar (T6-L5) vertebral compression fractures: Retrospective study of 111 patients with 140 fractured segments. J Turk Spinal Surg 30:199-205, 2019

16. Pflugmacher R, Beth P, Schroeder RJ, Schaser KD, Melcher I: Balloon kyphoplasty for the treatment of pathological fractures in the thoracic and lumbar spine caused by metastasis: Oneyear follow-up. Acta Radiol 48(1):89-95, 2007

17. Sciubba DM, Gokaslan ZL: Diagnosis and management of metastatic spine disease. Surg Oncol 15(3):141-151, 2006 
18. Selvaggi G, Scagliotti GV: Management of bone metastases in cancer: A review. Crit Rev Oncol Hematol 56(3):365-378, 2005

19. Siemionow K, Lieberman $\mathrm{IH}$ : Vertebral augmentation in osteoporotic and osteolytic fractures. Curr Opin Support Palliat Care 3(3):219-225, 2009
20. Uzunoglu I, Kaya I, Sucu HK, Kizmazoglu C, Sevin IE, Aydin HE, Yuceer N: Evaluation of incidentally detected pathology results of patients with vertebral fracture treated by vertebroplasty and kyphoplasty: A retrospective study. World Neurosurg 122: e639-e646, 2019

21. Zhang J, Shao HY, Xu SN, Huang YZ: Perforation of gastric wall by polymethylmethacrylate after percutaneous kyphoplasty: Case report and literature review. Turk Neurosurg 27(3):460463, 2017 